QIRT 10 in

\title{
Thickness or diffusivity measurements from front-face flash experiments using the TSR (thermographic signal reconstruction) approach \\ by Daniel L. Balageas
}

\author{
ONERA, Dept. of Composite Materials and Structures, BP 72, 92322 Châtillon, France \\ daniel.balageas@onera.fr
}

\begin{abstract}
We present the evaluation of possible quantitative applications of the TSR (thermographic signal reconstruction) technique in the simple case of the adiabatic homogeneous plate with the application of the measurement of thickness or diffusivity. Theoretical and experimental analysis of the identification is achieved, leading to recommendations in view of obtaining a precise and reliable evaluation of these parameters. In particular, the use of the first logarithmic derivative of the thermogram is recommended instead of the second logarithmic derivative. The method when applied to homogeneous, constant-thickness and quasi adiabatic samples allows to consider the front-face measurement as a possible flash diffusivity technique with an accuracy comparable to that of the classical rear-face diffusivity method of Parker.
\end{abstract}

\section{Introduction}

The TSR (Thermographic Signal Reconstruction) method proposed by Shepard [1-5], is well known and largely used in Non-Destructive Evaluation. The method, used for pulsed thermographic experiments, consists in:

- the fitting of the experimental log-log plot thermogram by a logarithmic polynomial:

$$
\ln (\Delta T)=a_{0}+a_{1} \ln (t)+a_{2}[\ln (t)]^{2} \ldots+a_{n}[\ln (t)]^{n}
$$

This fitting is particularly well suited for pulse heating. It is sometimes called Logarithmic Fitting Adiabatic (LFA) [6].

- the replacement of the sequence of temperature increase images, $\Delta T(i, j, t)$, by the series of $(n+1)$ images of the polynomial coefficients: $a_{0}(i, j), \ldots a_{n}(i, j)$. This operation allows a drastic reduction of the data amount. From this series of $(n+1)$ images it is then possible to reconstruct the full sequence of the experiment.

- the use for NDE purpose of the second derivative of the thermogram, $d^{2} \ln \left(\Delta T / \Delta T_{\infty}\right) / d \ln (F o)^{2}$, the derivation being achieved directly on the polynomial, then without increase of the temporal noise.

The TSR technique enhances the detectivity of defects i) by reduction of diffusion blurring and temporal noise, ii) improvement of the contrast between sound and defective areas, iii) production of images unaffected by a non uniform illumination, a non uniform absorptivity, or spurious radiations from environment including illumination sources and camera, and allows a high data compression, which makes possible the fusion of multiple sub-windowings of extended structures without artefacts [5]. These interesting performances have been recently corroborated by other groups using either the TSR technique alone, for instance to detect defects in GLARE [7], or in conjunction with the Pulse Phase Thermography approach, which is in this case considerably improved when detecting defects in honeycomb structures [8].

Despite an abundant literature, until now the use of the TSR technique remains relatively qualitative, if we except recent papers from Shepard $[4,9]$. As explained in the last reference, the situation is explicable since most of the applications of the method deal with NDE problems for which excellent detection performances are more important than precise properties measurements. The aims of the present work is the evaluation of possible quantitative applications of the TSR technique. The simpler case is presently analyzed: the adiabatic homogeneous plate with the application of an accurate measurement of the distribution of thickness or diffusivity in a plate. Although the TSR technique is essentially used for inspection of more complex structures presenting defects such as delaminations, debonds, voids..., this simple case is interesting because it allows to highlight the characteristic advantages of the technique and the way its quantitative use can be achieved. This approach is followed by Shepard in [4]. Here the approach is analogous but the exact analytical solutions are explicitely given for the first two derivatives expressed as functions of the Fourier number and the study is focused on the accuracy with which the $1^{\text {st }}$ and $2^{\text {nd }}$ logarithmic derivatives are approximated using the double derivation of the fitting polynomial and, finally, on the accuracy reached on the identified diffusivity or thickness.

\section{Analytical expressions of the thermogram and its derivatives in the case of an adiabatic homogeneous slab.}

The surface temperature of an adiabatic slab receiving a Dirac of energy is governed by the well-known formula:

$$
\Delta T / \Delta T_{\infty}=1+2 \sum_{1}^{\infty} e^{-n^{2} \pi^{2} F_{o}}
$$

where $F_{O}$ is the Fourier number of the slab, equal to $F_{O}=\kappa t / L^{2}$ and $\Delta T_{\infty}$ the final isothermal increase of temperature equal to $Q / \rho C L, Q$ being the density of energy (or fluence) entered in the slab and $\rho C$ the volume heat capacity. In the present case the temperature increase is depending on a unique variable, the Fourier number, and consequently on two physical parameters which are the diffusivity, $K$, and the thickness of the slab, $L$. Each parameter can be identified from the thermogram if the other is known. 
The first and second logarithmic derivatives of the thermogram are:

$$
\begin{aligned}
& d \log _{10}\left(\Delta T / \Delta T_{\infty}\right) / d \log _{10}(F o)=-2 \pi^{2} F o \sum_{1}^{\infty} n^{2} e^{-n^{2} \pi^{2} F o} /\left(1+2 \sum_{1}^{\infty} e^{-n^{2} \pi^{2} F o}\right) \\
& d^{2} \log _{10}\left(\Delta T / \Delta T_{\infty}\right) / d \log _{10}(F o)^{2}=-2 A \pi^{2} F_{o}\left\{\sum_{1}^{\infty} n^{2} e^{-n^{2} \pi^{2} F o} /\left(1+2 \sum_{1}^{\infty} e^{-n^{2} \pi^{2} F o}\right)\right. \\
& \quad-\pi^{2} F o\left[\sum_{1}^{\infty} n^{4} e^{-n^{2} \pi^{2} F o} /\left(1+2 \sum_{1}^{\infty} e^{-n^{2} \pi^{2} F o}\right)-2\left(\sum_{1}^{\infty} n^{2} e^{-n^{2} \pi^{2} F o}\right)^{2} /\left(1+2 \sum_{1}^{\infty} e^{-n^{2} \pi^{2} F o}\right)^{2}\right]
\end{aligned}
$$

Here the decimal logarithms $\left(\log (u)\right.$ or $\left.\log _{10}(u)\right)$ are used, being much more practical in physics than the natural logarithms $\left(\ln (u)\right.$ or $\left.\log _{e}(u)\right)$. This explains the existence of the constant $A$ in Eq. $(4): A=\log _{e}(u) / \log _{10}(u)=\log _{\mathrm{e}}(10)=2.30216$.

Figure 1 presents the pulse thermogram and the first and second logarithmic derivatives calculated following Eqs. (2-4). The thermogram can be split in three successive parts: a straight line of slope -0.5 in log-log domain, which corresponds to the semi-infinite medium regime, followed by a transition regime due to the finite thickness of the slab and characterized by a progressive decrease of the slope, and finally a final adiabatic plateau with an in-depth isothermal state of the slab. The $2^{\text {nd }}$ derivative presents a monopolar signature with a characteristic positive peak. In practice, especially if the material is a poor conductor, due to the existence of heat losses on both faces of the slab, the final plateau is replaced by a progressive return to the initial state $\left(\Delta T_{\infty}=0\right)$. In this case the $2^{\text {nd }}$ derivative peak is attenuated and followed by a decrease down to negative values. In the aforementioned more complex situations (structures with defects such as delaminations, debonding, voids, flat bottom holes), the $2^{\text {nd }}$ derivative signature becomes more complex, especially if the extent of the defect is laterally limited, inducing 2-D and 3-D heat diffusion which adds a negative peak followed by a second positive peak.

The Fourier number for which the $2^{\text {nd }}$ derivative peaks corresponds to a critical Fourier number Fo* $=1 / \pi$, for which the $1^{\text {st }}$ derivative presents an inflection and reaches half its minimum value,

$$
\left[d \log _{10}\left(\Delta T / \Delta T_{\infty}\right) / d \log _{10}(F o)\right]_{F^{*}}=-0.25
$$

The critical time or Fourier number $F_{O}{ }^{*}$ can be also determined on the thermogram itself as being the intersection of the extrapolated straight line of the semi-infinite medium regime with the extrapolated final plateau [10].

The peak value of the second derivative, using Eq. (4) is: $\left[\mathrm{d}^{2} \log _{10}\left(\Delta T / \Delta T_{\infty}\right) / \mathrm{d} \log _{10}\left(F_{O}\right)^{2}\right]_{F_{0^{*}}}=1.0904$. This peak value must depend on the reflection coefficient, $R$, due to the rear face of the slab, which can be considered as an interface between the slab material and the air. $R=\left(e_{i}-e_{j}\right) /\left(e_{i}+e_{j}\right), e_{i}$ and $e_{j}$ being the effusivities of the materials in contact at the interface. The air effusivity being negligible, in the present case $R=1$. In all other cases, encountered interfaces such as delaminations or contacts between different solid layers layers will be characterized by a smaller reflection coefficient, producing softer variations of the slope of the thermogram and consequently smaller peak values for the $2 \mathrm{nd}$ derivative. The finite lateral extents of encountered interfaces produced by defects leading to 3-D diffusion will have the same consequences on the peak value of the $2^{\text {nd }}$ derivative. They will produce lower peak values. In consequence, the value must be considered as the maximum maximorum of the possible values of this derivative.

The $2^{\text {nd }}$ derivative curve can be approximated with a very good accuracy by a Gaussian curve, as already described by Shepard et al. [4]. Here the Gaussian fitting leads to:

$$
d^{2} \log _{10}\left(\Delta T / \Delta T_{\infty}\right) / d \log _{10}(F o)^{2}=1.09 e^{-14.8\left[\log _{10}\left(F_{O} / F^{*}\right)\right]^{2}}=1.09 e^{-14.8\left[\log _{10}(\pi F o)\right]^{2}}
$$

The $R^{2}$ coefficient calculated on the Fo domain [0.05-2] is equal to 0.99981 which corresponds to an excellent fit. Figure 2 compares the exact $2^{\text {nd }}$ derivative curve and the approximate curve (Gaussian) and presents the corresponding residuals. They are smaller than $0.5 \%$ and localized at the feet of the Gaussian curve.

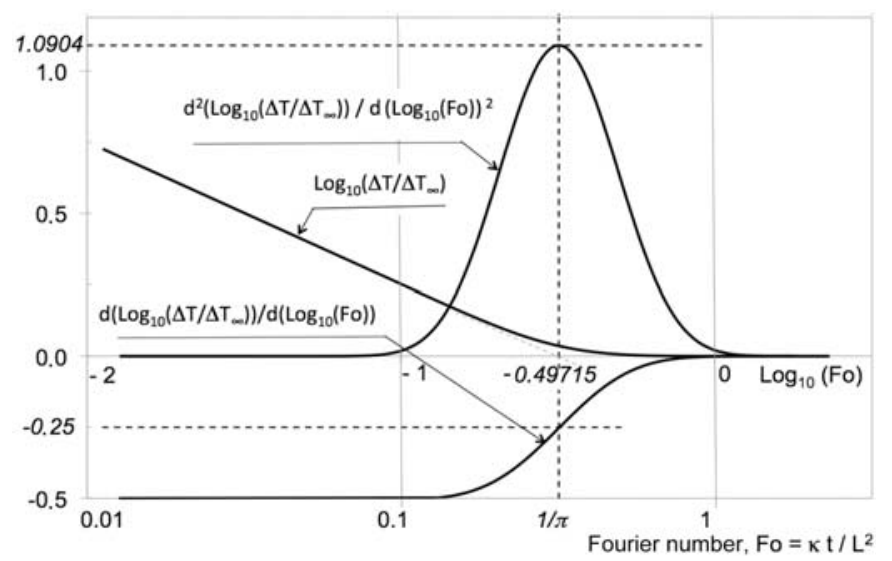

Figure 1. The pulse thermogram and its derivatives in log-log domain. Case of the homogeneous adiabatic plate.

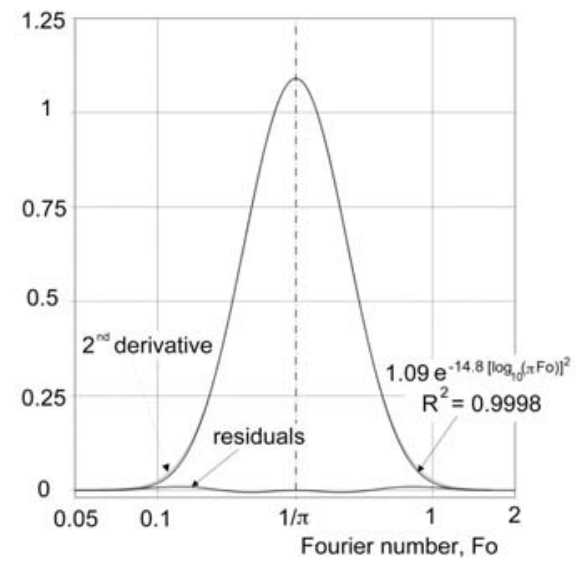

Figure 2. $2^{\text {nd }}$ derivative approximated by a Gaussian. Comparison to the exact solution and residuals. 


\section{Inverse problem: identification of the thickness or diffusivity}

Although the approximated expression of the $2^{\text {nd }}$ derivative, Eq. (6) could be used for solving the inverse problem of determining the diffusivity or the thickness (see Ref. [4]), we will focus on the simple way based on the determination of the critical Fourier number Fo* corresponding to both the inflexion point of the first derivative and the apex of the second derivative. Regarding the first derivative, this is a simple way of identification, similar to the one proposed by Parker et al. [11] for identifying the diffusivity from the half-rise time of the rear-face pulse thermogram. In both cases $\left(1^{\text {st }}\right.$ and $2^{\text {nd }}$ derivatives) no absolute temperature measurement is needed, but only the detection of the occurrence of these phenomena (inflection and peak). The weakness of the method is that it does not take into account all the information contained in the full curves $f^{\prime}(t)$ and $f^{\prime \prime}(t)$ and that it is not flexible. Nevertheless, for a uniform diffusivity and constant-thickness adiabatic slab, it is well adapted.

The $\kappa / L^{2}$ parameter is deduced from the critical Fourier number expression: $F O^{*}=\kappa t^{*} / L^{2}=1 / \pi$, leading to:

$$
\kappa / L^{2}=1 /\left(\pi t^{\star}\right)
$$

$t^{*}$ being deduced by one of the three possible methods already presented (intersection of the straight parts of the thermogram, half-rise point of the $1^{\text {st }}$ derivative, peak occurrence of the $2^{\text {nd }}$ derivative).

The TSR approach offers a solution for calculating the logarithmic derivatives of the thermogram: the fitting (least mean squares) of the thermogram by a logarithmic polynomial (expression [1]) and two successive derivations of this polynomial.

$$
\begin{aligned}
& \log _{10}(\Delta T)=P\left(\log _{10}(t)\right)=\sum_{0}^{n} a_{k}\left[\log _{10}(t)\right]^{k}, \\
& d \log _{10}(\Delta T) / d \log _{10}(t)=P^{\prime}\left(\log _{10}(t)\right)=\sum_{1}^{n} k a_{k}\left[\log _{10}(t)\right]^{k-1}, \\
& d^{2} \log _{10}(\Delta T) / d \log _{10}(t)^{2}=P^{\prime \prime}\left(\log _{10}(t)\right)=\sum_{2}^{n} k(k-1) a_{k}\left[\log _{10}(t)\right]^{k-2} .
\end{aligned}
$$

It is worth noting that no normalization of the thermogram is needed when using the first and second logarithmic derivatives. In other words, the possible non uniformity of the fluence or of the absorptivity/emissivity of the material have no influence on the logarithmic derivatives, at least if the characteristic length of their in-plane variations is several times larger than the slab thickness. This is the reason why in Eqs. (8-10) the thermogram is not normalized (we use $\Delta T$ instead of $\Delta T / \Delta T_{\infty}$ ).

To obtain a realistic estimate of the intrinsic accuracy of this TSR approach, it is necessary to start from a polynomial fitting of the exact theoretical thermogram, given by Equation (2):

$$
P\left(\log _{10}(t)\right)=\sum_{0}^{n} a_{k}\left[\log _{10}(t)\right]^{k} \approx \log _{10}\left(1+2 \sum_{1}^{\infty} e^{-n^{2} \pi^{2} \kappa t / L^{2}}\right),
$$

The possible influence of the sampling period, $\Delta F O$, if any, has not been studied and is supposed negligible. Here, $\Delta F o$ has been taken equal to 0.01 .

The comparison between the values of $\kappa / L^{2}(t)$ or Fo identified using the polynomials Eqs. (8-10) to the values used to calculate the thermogram (equation [2]) allows us to estimate the accuracy of the identification from the critical time $t^{*}$.

Figure 3 presents the so-fitted thermogram and its $1^{\text {st }}$ and $2^{\text {nd }}$ logarithmic derivatives. Two degrees have been considered for the polynomial $P$ : 6 and 9, and two domains of Fo for the fitting: [0.01-5] and [0.05-1.6]. Both parameters have a strong influence on the quality of the fitting, especially for the $2^{\text {nd }}$ derivative which appears logically very sensitive to them. The maximum and the width of the peak region are respectively too low and too large when $n=6$, but very near of the theoretical values for $n=9$. The use of the degree 6 combined with a wide fitting domain leads to a "wavy" curve for $P$ " which could make the detection of the second derivative "bump" questionable and difficult a fully automatic data reduction process.

These qualitative observations can be quantified by calculating the coefficient of determination $R^{2}$ of the polynomial $P, P^{\prime}$ and $P^{\prime \prime}$. This is possible since we know the exact expression of the thermogram and of its $1^{\text {st }}$ and $2^{\text {nd }}$ derivatives. For $n=9$ the three fitting polynomials are satisfying since their $R^{2}$ is not far from 1 . For $n=6, P^{\prime}$ is satisfactory but $P^{\prime \prime}$ cannot be used since the coefficient is negative which characterizes a very poor correlation between the data and the fitting. In all cases we see in Table 1 that each derivation of the fitting polynomial produces a degradation of the quality of the fitting.

\begin{tabular}{|c|c|c|c|c|}
\hline $\begin{array}{c}\text { Fo domain and number } \\
\text { of values considered } \\
\text { for the fitting }\end{array}$ & $\begin{array}{c}\text { Polynomial } \\
\text { degree, } n\end{array}$ & $\begin{array}{c}R^{2} \text { of } \\
\text { polynomial } P\end{array}$ & $\begin{array}{c}R^{2} \text { of } \\
\text { polynomial } P^{\prime}\end{array}$ & $\begin{array}{c}R^{2} \text { of } \\
\text { polynomial } P^{\prime \prime}\end{array}$ \\
\hline$\left[\begin{array}{c}{[0.01-5]} \\
500\end{array}\right.$ & 6 & 0.99912 & 0.8795 & $<0$ \\
\hline$[0.05-1.6]$ & 6 & 0.99985 & 0.9373 & $<0$ \\
\hline 156 & 9 & 0.99997 & 0.9566 & 0.3959 \\
\hline & 9 & 0.99999 & 0.9992 & 0.9393 \\
\hline
\end{tabular}

Table 1. Degradation of the coefficient $R^{2}$ with the successive derivations of the polynomial $P$ fitting the exact analytical expression of the thermogram, Eq. (2). The $R^{2}$ coefficients of the derivative polynomials are calculated by comparison to the exact analytical expressions of the logarithmic derivatives of the thermogram, Eqs. $(3,4)$. 


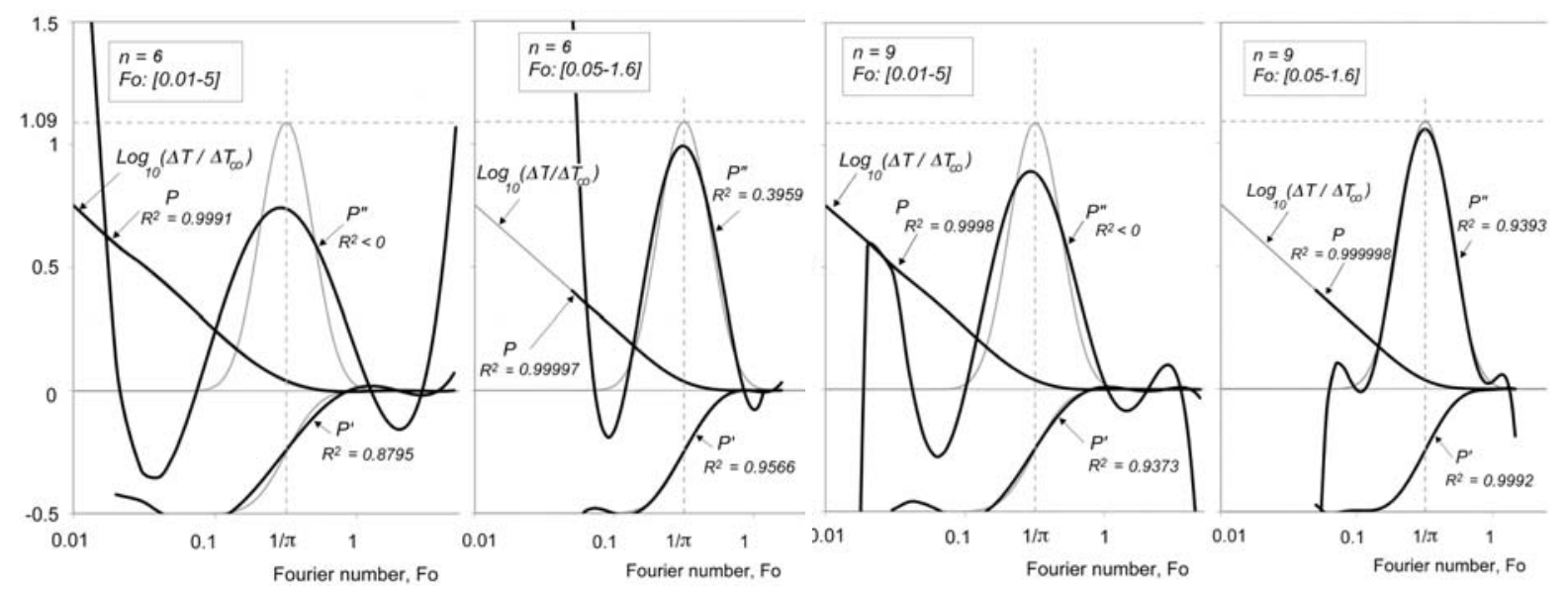

Figure 3. Fitting by a polynomial of the theoretical thermogram given by Eq.(2). Combined influences of the degree of the polynomial and of the extent of the Fourier domain considered for the fitting.

\begin{tabular}{|c|c|c|c|c|}
\hline $\begin{array}{c}\text { Fo domain } \\
\text { for fitting }\end{array}$ & $\begin{array}{c}\text { Polynomial } \\
\text { degree }\end{array}$ & $\begin{array}{c}F o\left(f^{\prime}=0.25\right) \\
\text { abs. value (accuracy) }\end{array}$ & $\begin{array}{c}F o\left(f^{\prime \prime}{ }_{\max }\right) \\
\text { abs. value (accuracy) }\end{array}$ & $\begin{array}{c}f^{\prime \prime}{ }_{\max } \\
\text { abs. value (accuracy) }\end{array}$ \\
\hline Exact solution & & 0.3183 & 0,3183 & 1.0904 \\
\hline \multirow{2}{*}[0.01-5]{} & 6 & $0.309(-2,9 \%)$ & $0.291(-8.6 \%)$ & $0,7429(-31,9 \%)$ \\
\cline { 2 - 5 } & 9 & $0.312(-2,0 \%)$ & $0.295(-7.3 \%)$ & $0.8915(-18.2 \%)$ \\
\hline \multirow{2}{*}[0.05-1,6]{} & 6 & $0.3168(-0.5 \%)$ & $0.3122(-1.9 \%)$ & $0.9913(-9.1 \%)$ \\
\cline { 2 - 5 } & 9 & $0.3181(-0.1 \%)$ & $0.3175(-0.3 \%)$ & $1.0575(-3.0 \%)$ \\
\hline
\end{tabular}
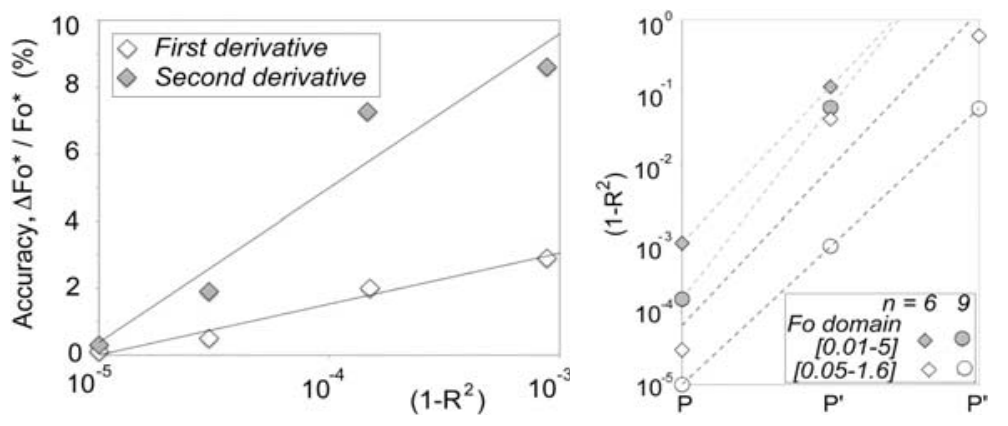

Table 2. Accuracy i) of the identified values of the critical Fourier number, Fo*, deduced from the analysis of the $P^{\prime}$ and $P$ ", ii) of the peak value of $P^{\prime \prime}$.

The accuracy of the identification of the critical Fourier number, $F_{O^{*}}$, is presented in Table 2. The accuracy on the value of the peak of the $2^{\text {nd }}$ derivative is also given. It is worth noting that the accuracy of the identification is always satisfying when using the $1^{\text {st }}$ derivative, which is not the case for the $2^{\text {nd }}$ derivative.

Figure 4a shows that the accuracy of the identification is correlated to the determination coefficient $R^{2}$ and Figure $4 \mathrm{~b}$ that each successive derivations of the fitting polynomial $\mathrm{P}$ produces a degradation of the quality of the fitting, characterized by a gap to 1 of the coefficient $R^{2}$ which increases by roughly two orders of magnitude.

\section{Conclusions drawn from the theoretical analysis}

The thermogram and its derivatives are the exact analytical solutions and they are not affected by any noise. The accuracies obtained can be considered as intrinsic of the TSR method. In practice, they can be affected by noise. This will be studied in the experimental part.

The accuracy of the identification of the parameter $\kappa / L^{2}$ from the occurrence time $t^{*}$ of the peak value of the $2^{\text {nd }}$ logarithmic derivative approximated by the $2^{\text {nd }}$ derivative of the fitting polynomial of the full thermogram, $P$ ", is dependent on the degree of the polynomial which must be high $(n>6)$ in the present case, and highly dependent on the fitting time domain (see Table 2). If the fitting is made from the full thermogram (including the initial straight line of slope -0.5 and the final adiabatic plateau) even a polynomial degree as high as 9 leads to errors higher than $5 \%$ (and sometimes much more) on the occurrence time $t^{*}$ and consequently on the identified parameter $\kappa / L^{2}$.

Using the occurrence of the half-rise of the first derivative seems more reliable and precise since the errors are less than $3 \%$ for the whole cases analyzed in Table 2.

Finally, the determination coefficient $R^{2}$ is a good criteria to judge of the reliability of the fitting and of the accuracy of the identification. It could be used for optimizing the choice of the parameters (polynomial degree and size of the domain fitted). 


\section{Experimental validation}

The proposed method has been verified by the measurement of the diffusivity of a metallic slab of known and uniform thickness. The sample was illuminated by two flash lamps (Elinchrom $6000 \mathrm{~J}$ ) located symmetrically in front of the sample at a distance of $60 \mathrm{~cm}$. The duration of the pulse is estimated to be less than $4 \mathrm{~ms}$, and the fluence $0.2 \mathrm{~J} \mathrm{~cm}^{-2}$. The temperature of the front-face or of the rear face was monitored by a long-wave infrared camera Jade from Cedip Company equipped with a LW 2.0 lens. The image frequency was $200 \mathrm{~Hz}$. The sample was a $10 \mathrm{~cm} \times 10 \mathrm{~cm}, 10 \mathrm{~mm}$-thick plate of Dural. Such a sample satisfies perfectly the assumption of adiabaticity which is assumed in the theory presented here.

\subsection{Experimental thermograms}

The plate was tested with and without a $15 \mu \mathrm{m}$-thick carbon-black coating on the front face. The experimental thermograms are presented in Figure 5. For each case, two thermograms are presented: in grey the temperature evolution of a single pixel and in black the mean temperature of a disk-shape region, centred on this single pixel and containing 26 pixels. This second thermogram has a better signal-to-noise ratio.

The uncoated sample leads to temperature increases which are $51 \%$ of the ones of the coated sample. The coating has an influence on the shape of the thermogram up to $0.1 \mathrm{~s}$, a time higher that the usual period (roughly evaluated to four times the pulse duration, here $0.014 \mathrm{~s}$ ) at the end of which the finite duration effect becomes negligible, allowing the thermogram to follow the $1 / \sqrt{t}$ law. This is due to the coating effusivity, much lower than the metal effusivity. Thus, at the very beginning, the front-face temperature is higher than that of the uncoated metallic plate. The thermogram is in a transition phase, progressively more and more influenced by the metallic substrate. This two-layer effect is not present in the uncoated sample thermogram which follows the $1 / \sqrt{t}$ law after $0.01 \mathrm{~s}$. So, the data reduction must consider just the part of the thermogram after $0.1 \mathrm{~s}$ in the case of the coated sample, the only case considered due to the poor signal-to-noise ratio of the uncoated sample thermogram and that 3-D internal heat transfer due to a non uniform absorptivity of the Dural sample can be detected in the transition phase and in the final plateau.
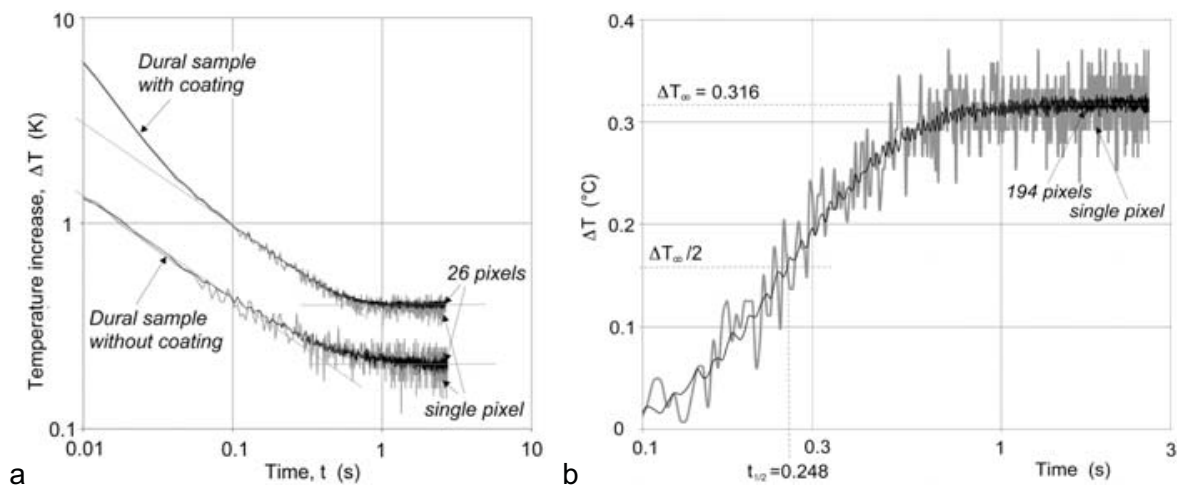

Figure $5 . \quad$ Experimental thermograms obtained with with a 10-mm thick Dural plate. a) Front-face pulsed thermograms with and without a carbon-black coating; b) Rear-face thermograms on the same sample and determination of the diffusivity from the half-rise time of the mean thermogram of a zone comprising 194 pixels. The fluence is the same as for the front-face measurements.

\subsection{Results of the logarithmic polynomial fitting and of the diffusivity identification}

The TSR technique is applied to both single-pixel and 26-pixel thermograms to study the possible influence of the noise on the results. Like in the theoretical study, the fitting is conducted with polynomials of degree 6 and 9 , and for two time domains: i) the first domain considered $[0.1 \mathrm{~s}-2.65 \mathrm{~s}]$ is be the entire thermogram, excepted the part before $t=0.1 \mathrm{~s}$ for the reason given previously, ii) a second domain, less wide, [0.3 s-1.5 s] is also considered. In total, there will be eight different cases analyzed ( 2 thermograms $\times 2$ polynomial degrees $\times 2$ time domains). No detailed discussion will be given about each of the eight presented cases, but a synthetic analysis will be given in the following section.

As seen in Figure $5 a$, the noise of a single pixel is important. For instance at the end of the experiment (adiabatic plateau) the signal to noise-ratio, taken as the ratio of the mean value of the plateau to the standard deviation for the plateau region, is only equal to 14 .

Figure 6 (first line) presents the results of the fitting of the thermogram of a single pixel for four different combinations of the two parameters: degrees of the polynomial and time domain considered for the fitting. In each case the graph presents the thermogram, the polynomials $P, P^{\prime}$ and $P^{\prime \prime}$ and the critical time, $t^{*}$, determined following the three procedures: i) intersection of the extrapolated straight line corresponding to the $1 / \sqrt{t}$ region (obtained by a linear fitting) with the extrapolated plateau, ii) abscissa of the half-rise $P^{\prime}$ curve, iii) abscissa of the peak of the $2^{\text {nd }}$ derivative, $P$ "'

All the parameters identified from the thermogram and from the logarithmic polynomials $P^{\prime}$ and $P^{\prime}$ are exhaustively presented in Table 3 . These parameters are: i) the critical time, $t^{\star}$ (three different evaluations), ii) the diffusivity, $\kappa$, which is deduced using Eq. (7) and alternatively the thickness, $L$, iii) the difference with the diffusivity deduced on the rear face of the same sample from the Parker's formula, value which can be considered as the reference, iv) the accuracy on the identified thickness which is half that of the diffusivity, v) as a complementary information: the parameter $R^{2}$ for the polynomial $P$.

The thermogram of the rear-face is presented in Figure $5 \mathrm{~b}$. This thermogram is obtained in the same conditions of illumination. For a single pixel, the signal-to noise ratio is very low: 13. In effect, the fluence is relatively low and corresponds to classical non destructive tests on large structures. In classical thermophysical characterization conditions, specially dedicated 
benches deliver higher fluences. Nevertheless, it is interesting to compare front-face and rear-face measurement obtained with the same illuminator, camera and sample. For a good accuracy, the considered thermogram is the mean temperature calculated on a zone of the image comprising 194 pixels. In this case the signal-to-noise ratio is satisfactory: 63. The diffusivity is calculated from the half-rise time Parker's formula, recommended in the present case since there is no noticeable heat losses:

$$
\kappa=0.139 L^{2} / t_{1 / 2}
$$

Finally, the polynomials obtained in the case of the 26 pixel thermogram for the four different combinations of the two parameters: degrees of the polynomial and time domain considered for the fitting, are presented in Figure 6 , second line.

\begin{tabular}{|c|c|c|c|c|c|c|c|c|c|c|c|}
\hline \multirow[b]{2}{*}{$\begin{array}{l}\text { type } \\
\text { of } \\
\text { meas. }\end{array}$} & \multirow[b]{2}{*}{$\begin{array}{l}\text { therm- } \\
\text { ogram }\end{array}$} & \multirow[b]{2}{*}{$\begin{array}{l}\text { time domain for } \\
\text { the fitting by } \\
\text { polynomial } P\end{array}$} & \multirow[b]{2}{*}{$\begin{array}{c}\text { Degree } \\
\text { n of } \\
P\end{array}$} & \multirow[b]{2}{*}{$\begin{array}{c}\text { source } \\
\text { of the } \\
\text { information }\end{array}$} & \multirow[b]{2}{*}{$\begin{array}{c}\text { coeff. } \\
\mathrm{R}^{2} \\
\text { of } P\end{array}$} & \multirow[b]{2}{*}{$\begin{array}{l}S / N \\
(1 \sigma)\end{array}$} & \multirow[b]{2}{*}{$\begin{array}{l}\text { critical } \\
\text { time } \\
t^{\star}(\mathrm{s})\end{array}$} & \multicolumn{2}{|c|}{ identified diffusivity ${ }^{2}$} & \multicolumn{2}{|c|}{ identified thickness ${ }^{2}$} \\
\hline & & & & & & & & $\begin{array}{c}k=L^{2} / \pi t^{*} \\
\mathrm{~m}^{2} \mathrm{~s}^{-1} \times 10^{5}\end{array}$ & $\begin{array}{l}\text { accuracy } \\
\text { (reference: } \\
\text { Parker) }\end{array}$ & $\begin{array}{c}L= \\
\left(\pi t^{*} k_{\text {Parker }}\right)^{1 / 2} \\
\mathrm{~cm}\end{array}$ & accuracy \\
\hline \multirow{26}{*}{$\begin{array}{l}\text { front } \\
\text { face }\end{array}$} & \multirow{13}{*}{$\begin{array}{c}\text { single } \\
\text { pixel }\end{array}$} & \multirow{7}{*}[0.10-2.65]{} & & thermogramme $^{1}$ & & 14 & 0.607 & 5.24 & $-5.7 \%$ & 1,030 & $3,0 \%$ \\
\hline & & & \multirow{3}{*}{6} & polynomial $P$ & 0.870 & & & & & & \\
\hline & & & & $1^{\text {st }}$ derivative $\left(P^{\prime}\right)$ & & & 0.586 & 5.43 & $-2.3 \%$ & 1.012 & $1.2 \%$ \\
\hline & & & & $2^{\text {nd }}$ derivative $\left(P^{\prime \prime}\right)$ & & & 0.569 & 5.59 & $0.62 \%$ & 0.997 & $-0.31 \%$ \\
\hline & & & \multirow{3}{*}{9} & polynomial $P$ & 0.87 & & & & & & \\
\hline & & & & $1^{\text {st }}$ derivative $\left(P^{\prime}\right)$ & & & 0.616 & 5.17 & $-7.1 \%$ & 1.037 & $3.7 \%$ \\
\hline & & & & $2^{\text {nd }}$ derivative $\left(P^{\prime \prime}\right)$ & & & 0.659 & 4.83 & $-13.1 \%$ & 1.073 & $7.3 \%$ \\
\hline & & \multirow{6}{*}[0.30-1.50]{} & \multirow{3}{*}{6} & polynomial $P$ & 0.648 & & & & & & \\
\hline & & & & $1^{\text {st }}$ derivative $\left(P^{\prime}\right)$ & & & 0.628 & 5.07 & $-8.8 \%$ & 1.047 & $4.7 \%$ \\
\hline & & & & $2^{\text {nd }}$ derivative $\left(P^{\prime \prime}\right)$ & & & 0,672 & 4.74 & $-14.8 \%$ & 1.083 & $8.3 \%$ \\
\hline & & & \multirow{3}{*}{9} & polynomial $P$ & 0.65 & & & & & & \\
\hline & & & & $1^{\text {st }}$ derivative $\left(P^{\prime}\right)$ & & & 0.653 & 4.87 & $-12.3 \%$ & 1.068 & $6.8 \%$ \\
\hline & & & & $2^{\text {nd }}$ derivative $\left(P^{\prime \prime}\right)$ & & & 0.720 & 4.42 & $-20.5 \%$ & 1.121 & $12.1 \%$ \\
\hline & \multirow{13}{*}{$\begin{array}{l}\text { means } \\
\text { of } \\
26 \\
\text { pixels }\end{array}$} & & & thermogramme ${ }^{1}$ & & 46 & 0.578 & 5.51 & $-1.0 \%$ & 1.005 & $0.48 \%$ \\
\hline & & \multirow{6}{*}{ [0.10 - 2.65] } & \multirow{3}{*}{6} & polynomial $P$ & 0.99 & & & & & & \\
\hline & & & & $1^{\text {st }}$ derivative $\left(P^{\prime}\right)$ & & & 0.581 & 5.48 & $-1.5 \%$ & 1.007 & $0.74 \%$ \\
\hline & & & & $2^{\text {nd }}$ derivative $\left(P^{\prime \prime}\right)$ & & & 0.578 & 5.51 & $-1.0 \%$ & 1.005 & $0.48 \%$ \\
\hline & & & \multirow{3}{*}{9} & polynomial $P$ & 0.99 & & & & & & \\
\hline & & & & $1^{\text {st }}$ derivative $\left(P^{\prime}\right)$ & & & 0.592 & 5.38 & $-3.3 \%$ & 1.017 & $1.7 \%$ \\
\hline & & & & $2^{\text {nd }}$ derivative $\left(P^{\prime \prime}\right)$ & & & 0.621 & 5.13 & $-7.8 \%$ & 1.041 & $4.1 \%$ \\
\hline & & \multirow{6}{*}[0.30-1.50]{} & \multirow{3}{*}{6} & polynomial $P$ & 0.95 & & & & & & \\
\hline & & & & $1^{\text {st }}$ derivative $\left(P^{\prime}\right)$ & & & 0.568 & 5.60 & $0.79 \%$ & 0.996 & $-0.39 \%$ \\
\hline & & & & $2^{\text {nd }}$ derivative $\left(P^{\prime \prime}\right)$ & & & 0.463 & 6.87 & $23.7 \%$ & 0.899 & $-10.1 \%$ \\
\hline & & & \multirow{3}{*}{9} & polynomial $P$ & 0.95 & & & & & & \\
\hline & & & & $1^{\text {st }}$ derivative $\left(P^{\prime}\right)$ & & & 0.571 & 5.57 & $0.26 \%$ & 0.999 & $-0.13 \%$ \\
\hline & & & & $2^{\text {nd }}$ derivative $\left(P^{\prime \prime}\right)$ & & & 0.491 & 6.48 & $16.6 \%$ & 0.926 & $-7.4 \%$ \\
\hline \multicolumn{7}{|c|}{$\begin{array}{l}\text { Intersection of the extrapolated initial straight line with the final adiabatic plateau } \\
2 \text { Alternate identifications following which parameter is initially known }\end{array}$} & $t_{1 / 2}$ & $\begin{array}{c}k_{\text {Parker }}= \\
0.139 L^{2} / t_{1 / 2}\end{array}$ & & & \\
\hline $\begin{array}{l}\text { rear } \\
\text { face }\end{array}$ & $\begin{array}{l}\text { means } \\
\text { of } 194 \\
\text { pixels }\end{array}$ & & & $\begin{array}{l}\text { locally } \\
\text { fitted } \\
\text { thermogram }\end{array}$ & & 62 & 0.250 & $5.56 \mathrm{E}-05$ & & & \\
\hline
\end{tabular}

Table 3. Diffusivity of the coated Dural sample, deduced from the thermo-gram and from the logarithmic polynomials $P^{\prime}$ and $P$ ", and comparison to the rear-face determination using Parker's formula.

\subsection{Analysis of the experimental results}

The results of the real thermograms are notably different from those of the theoretical analysis (sections 3 and 4 ). The graphs presented in figures 6 show that the results obtained with the lower polynomial degree $(n=6)$ are better than those obtained with the higher degree tested, $n=9$ : i) the shapes of the derivatives curves are closer to those of the theoretical curves, ii) the value of the $2^{\text {nd }}$ derivative peak and the width of the Gaussian shape are closer to the theoretical values. The explanation lies in the fact that the theory supposes no noise and just a perfect 1-D conduction process. When considering real thermograms, with noise and possible 3-D conduction due to non-uniform illumination and non-uniform optical properties of the sample, the increase of the polynomial degree (the degree of freedom) gives more influence to the noise and more flexibility to be contaminated by parasitic effects. These phenomena increase with the noise, as demonstrated by comparing the results from the 26 pixel thermogram $(S / N=46)$ to those of the single pixel thermogram $(S / N=14)$.

The beneficial influence of a decrease of the size of the temporal domain considered for the fitting no more exists. A reverse influence is in fact shown for both degrees $n=6$ and $n=9$. The explanation could be that the quantity of information coming from the thermal phenomena is decreasing when reducing the time domain contrarily to the noise information which remains constant. This is quantified by a decrease of the $R^{2}$ coefficient of the polynomial $P$ when the size of the time domain decreases.

An important point is that the first derivative is less sensitive to the noise than the second derivative, in particular in its half-rise region, which is important since the critical time $t^{*}$ is inside this part of the curve.

From a quantitative point of view, the quality of the identification methods can be assessed by comparison of the identified diffusivities to the diffusivity measured by the rear-face experiment on the same sample, using the Parker's formula. We can state that: i) the method which consists to determine the intersection of the two straight parts of the thermogram ( $1 / \sqrt{t}$ part and final plateau) leads to pretty good results (accuracy of $-1 \%$ and $-6 \%$ ); ii) the TSR method using 


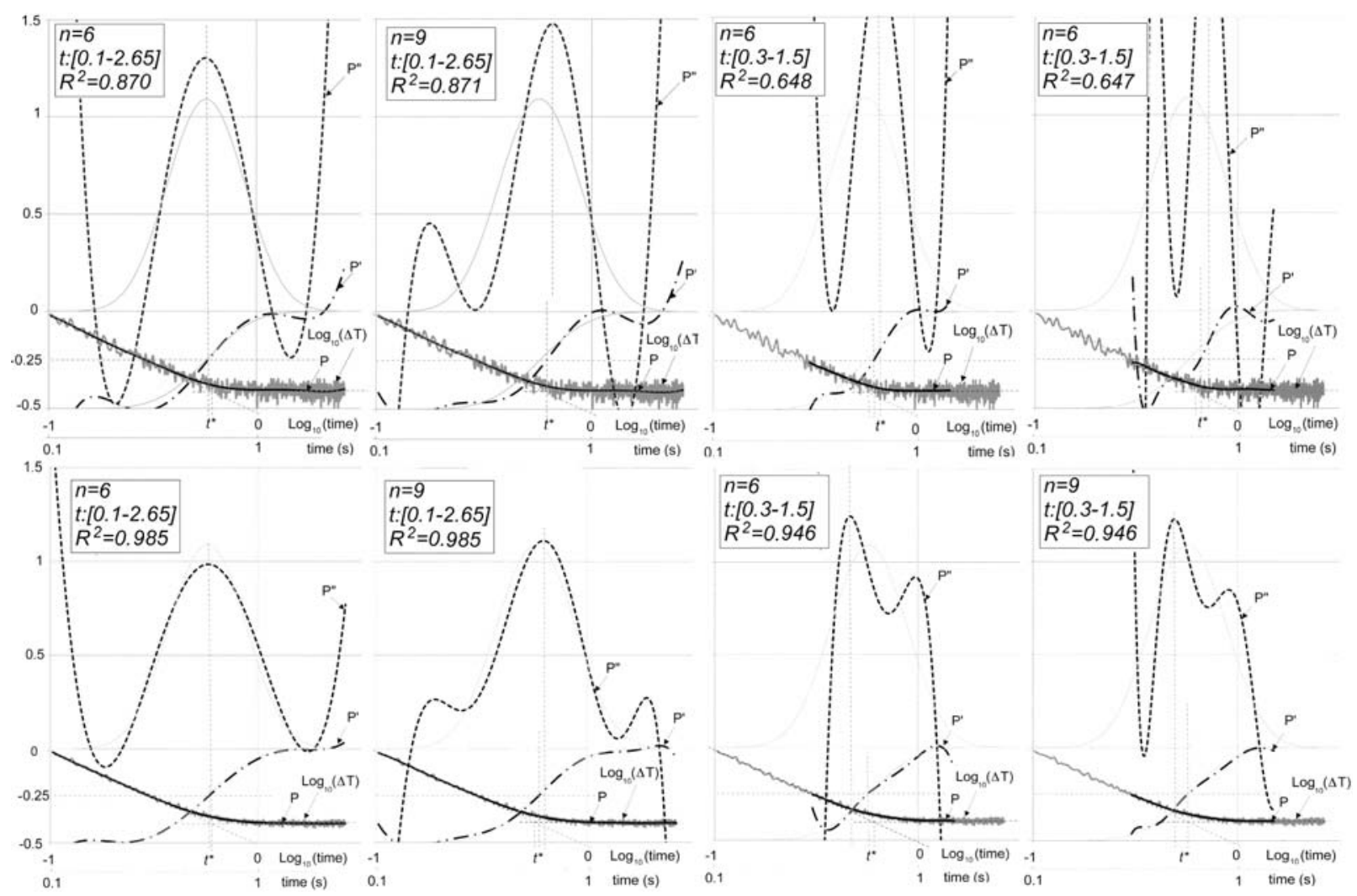

Figure 6. Dural sample with carbon-black coating. First line: fitting of the single pixel thermogram for the time domain $[0.1 \mathrm{~s}-2.65 \mathrm{~s}]$ and $[0.3 \mathrm{~s}-1.5 \mathrm{~s}$ ], using logarithmic polynomials $P$ of degrees 6 and 9; Second line: fitting of the mean thermogram of a 26 pixel zone for the same time domains and polynomial degrees.

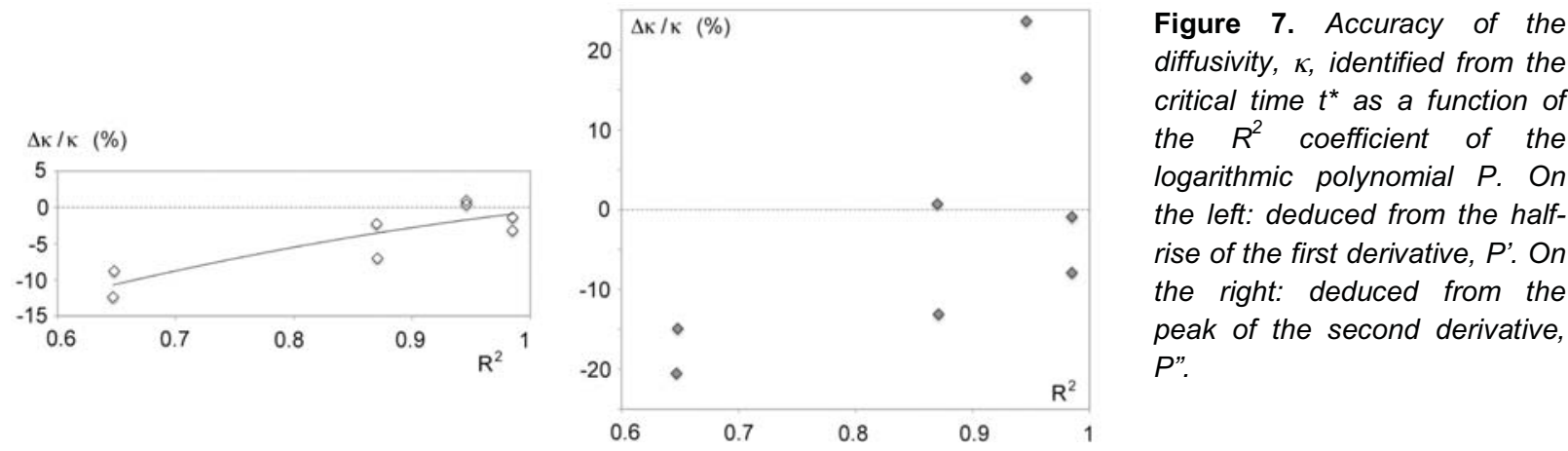

the first derivative and a low noise thermogram leads to very good results (errors between $-3 \%$ and $1 \%$ ) and fair results with the noisy thermogram (errors between $-2 \%$ and $-12 \%$ ); iii) the TSR method using the second derivative leads to erratic and sometimes bad results with both thermograms (errors between $-21 \%$ and $+17 \%$ ).

In practice, the question is: how to select the best combination of polynomial degree and time domain for the fitting? The answer could be found in the $R^{2}$ coefficient. Figure 7 presents the errors on the diffusivity as a function of the $R^{2}$ coefficient of the polynomial fitting the thermogram, $P$, whatever be the values of parameters: noise, polynomial degree, time domain. In figure 7left, the accuracy of the diffusivity determination is rather well correlated with the $R^{2}$ parameter for the values deduced from the occurrence time of the half-rise point of the $1^{\text {st }}$ derivative. For $R^{2}$ coefficient near of 1 the quality of the results are very precise (a few percents). For the diffusivity deduced from the occurrence of the peak of the second derivative, the scatter on the accuracies is very large and no correlation can be deduced. The adjunction of the third parameter, the noise, makes the things much complicated, impeding to confirm the results of the numerical simulations.

From this last analysis we could deduce a practical strategy to optimize the conditions of use of the TSR method in the specific application considered here: i) to use the first derivative first, ii) to try different fittings with various polynomial degrees and different time domains, iii) to choose the combination leading to the higher $\mathrm{R}^{2}$ coefficient, the closer possible of unity. The use the second derivative, in the present case is not recommended. 


\section{Conclusion and perspectives}

This quantitative study of the Thermographic Signal Reconstruction (TSR) method is strictly limited to the case of a front-face measurement in view of determining alternatively the diffusivity or the thickness of a plate shaped sample in adiabatic conditions. Consequently, the conclusions resulting from the theoretical analysis and from the application of the method to the measurement of the diffusivity of a Dural sample are only valid for this specific case.

The theoretical study is based on the exact analytical expressions of the thermogram and of its logarithmic derivatives. The TSR approach is applied to the exact analytical thermogram and the resulting $1^{\text {st }}$ and $2^{\text {nd }}$ logarithmic derivative polynomials are compared to the exact solutions. The influence of the degree of the polynomials and of the time domain considered for the polynomial fitting has been studied, showing a sensitivity of the shape of the $2^{\text {nd }}$ derivative making its quantitative use (identification of the diffusivity or thickness) somewhat difficult in practice. On the contrary, the use of the $1^{\text {st }}$ derivative appears more reliable and precise. The calculation of the determination coefficient $R^{2}$ shows that the successive derivations of the fitting polynomial produce a degradation, which may become inacceptable in many cases for a quantitative use of the $2^{\text {nd }}$ derivative.

The theoretical study has been completed by the analysis of thermographic experiments. Two front-face pulsed thermograms obtained on a plate of Duraluminum, characterized by different signal-to-noise ratios, have been analyzed and completed by a rear-face experiment leading to a good estimate of the diffusivity of the sample. The influence of the degrees of the polynomials and of the extent of the time domain considered have been analyzed. The conclusions of the experimental study are not so clear as the ones of the theoretical analysis, highlighting the strong influence of the noise, not considered in the theory. Nevertheless, the results recommend the use of the half-rise point of the $1^{\text {st }}$ logarithmic derivative polynomial and confirm in this case the correlation between the accuracy of the identification and the $R^{2}$ coefficient of the fitting polynomial.

It would be interesting to complete this study of the adiabatic plate by evaluating and comparing the accuracy reached by the approach presented by Shepard [4], which, starting from the fact that the $2^{\text {nd }}$ derivative is very close to a Gaussian, fit the thermogram by a function resulting from the double integration of a Gaussian function.

Due to the fact that the variance of the noise on $\log _{10}[\Delta T(t)]$, at time $t$, for a signal to noise ratio not too low, is equal to $\sigma^{2} / \Delta T(t)$, another improvement of the method could be, for the estimation of the polynomial coefficients, $a_{i}$, the replacement of the linear least-square method by a modified method in which the squares of the differences would be divided by their variance approximated by $\sigma^{2} / \Delta T(t)$ (private communication from Denis Maillet and ref. [12]).

In the future, it is envisaged to apply the methodology of the quantitative approach followed in this study to more complex situations, in particular to plate with heat losses, samples with a non-uniform in-plane diffusivity or a non-uniform thickness, layered structures with resistive defects or interfaces...

\section{REFERENCES}

[1] Shepard S.M., Ahmed T., Rubadeux B.A., Wang D., Lhota J.R., "Synthetic processing of pulsed thermographic data for inspection of turbine components", Insight, Vol. 43, No 9, Sept. 2001, British Inst. of NDT, pp. 587-589.

[2] Shepard S.M., Lhota J.R., Rubadeux B.A., Wang D., Ahmed T., "Reconstruction and enhancement of active thermographic image sequences", Optical Engineering, Vol. 42, No 5, May 2003, pp. 1337-1342.

[3] Shepard S.M., Hou Y.L., Ahmed T., Lhota J.R., "Reference-free Interpretation of Flash Thermography Data", Insight, Volume 48, No. 5, British Institute of NDT, May 2006, pp. 298-307.

[4] Shepard S.M., Hou Y.L., Lhota J.R., Golden J.M., "Automated Processing of Thermographic Derivatives for Quality Assurance", Optical Engineering, Vol. 46, No. 5, SPIE, May 2007, pp. 510081-6.

[5] Shepard S.M., "Flash Thermography of Aerospace Composites ", Fourth Pan American Conference for NDT, October 2007, Buenos Aires, Argentina - Open archives on http://www.ndt.net/article/panndt2007/papers/132.pdf

[6] Vavilov, V., Nondestructive Testing Handbook, Vol. 5, Book 1: Thermal/infrared testing, Spektr Publishing House, Moscow, 2009.

[7] Ibarra-Castanedo C. et al., "Delamination detection and impact damage assessment of GLARE by active thermography", Intern. J. of Materials \& Product Technology, Manuscript Reference No.: IJMPT-4NDT-01, in press, 2009.

[8] Ibarra-Castanedo C. et al., "Comparative study of active thermography techniques for the nondestructive evaluation of honeycomb structures", Research in Nondestructive Evaluation - RNDE, Taylor \& Francis, Vol. 20, 2009, pp.1-31.

[9] Shepard S.M., Lhota J.R., Ahmed T., "Measurement limits in flash thermography", SPIE Thermosense XXXIth Conference, Proc. SPIE Conf. Vol. 7299, 2009.

[10] Balageas D., "Thermal diffusivity measurement by pulsed methods", High Temperatures-High Pressures, vol. 21, pp 85-96, 1989.

[11] Parker W.J., Jenkins R.J., Butler C.P., Abbott G.L., "Flash method of determining thermal diffusivity, heat capacity and thermal conductivity", J. Applied Physics, Vol. 32, pp. 1679-1684, Sept. 1961.

[12] Petit D., Maillet D., "Techniques inverses et estimation de paramètres", Les Techniques de l'Ingénieur, Section AF 4515, pp. 118, and AF 4516, pp. 1-24, Paris, janvier 2008. 